\title{
Clinico-therapeutic management of osteoradionecrosis: A literature review and update
}

\author{
Javier Silvestre-Rangil ${ }^{1}$, Francisco-Javier Silvestre ${ }^{2}$
}

\author{
${ }^{1}$ Dental surgeon in private practice (Valencia, Spain) \\ ${ }^{2}$ Assistant Professor of Stomatology, Valencia University Medical and Dental School. Head of the Stomatology Unit, Dr. Peset \\ University Hospital. Valencia (Spain)
}

Correspondence:

Hospital Universitario Dr. Peset,

Consultas Externas,

C/ Juan de Garay s/n,

46017-Valencia (Spain),

francisco.silvestre@uv.es Silvestre-Rangil J, Silvestre FJ. Clinico-therapeutic management of os-
teoradionecrosis: A literature review and update. Med Oral Patol Oral Cir
Bucal. 2011 Nov 1;16 (7):e900-4.
http://www.medicinaoral.com/medoralfree01/v16i7/medoralv16i7p900.pdf

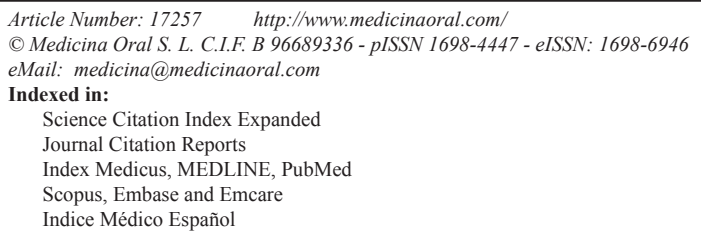

\begin{abstract}
In the management of head and neck cancer, radiotherapy is usually a coadjuvant to surgery, or is applied on a palliative basis. The most important complication of such radiotherapy is osteoradionecrosis, which manifests as an area of exposed necrotic bone in the maxillae or mandible that fails to heal during at least three months. In most cases osteoradionecrosis gradually progresses, becoming more extensive and painful, and its late manifestations comprise infection and pathological fracture.

The present study provides a literature review and update on the risk factors underlying osteoradionecrosis, its clinical and diagnostic particulars, prevention, and most widely accepted treatment options, as well as new possibilities relating to clinical management of the disorder. Lastly, a new early management protocol is proposed based on the current consensus criteria relating to maxillary osteonecrosis secondary to treatment with bisphosphonates, together with the adoption of new therapies supported by increased levels of evidence.
\end{abstract}

Key words: Osteoradionecrosis, risk factors, clinical manifestations, diagnosis, prevention, treatment.

\section{Introduction}

Osteoradionecrosis (ORN) of the maxillae is the most serious and important complication of radiotherapy (RT) administered in patients for head and neck tumors. RT is usually prescribed as adjuvant therapy following oncological surgery; however, it can be administered together with chemotherapy or as palliative treatment (1).

Osteoradionecrosis is defined as an area of exposed necrotic bone in one or more locations of the maxillary or mandibular alveolar process, with failure to heal during at least three months. ORN can develop spontaneously in one-third of all cases, but usually manifests after tooth extraction, after oral surgery, or due to irritation caused by poorly fitting dentures. In most cases the condition is chronic, progresses gradually and becomes more extensive and painful $(2,3)$.

Marx (3) defined ORN as resulting from the effects of RT, i.e., hypovascularization, hypoxia and hypocellularity - these circumstances in turn adversely affecting tissue repair capacity. At present a fibro-atrophic mechanism has been suggested, characterized by alterations in bone metabolism and the formation of free radicals, 
inflammation and microvascular thrombosis that evolve towards necrosis of the affected tissues (4).

Clinically, ORN remains latent and without symptoms until the lesions are visually detected or the patient begins to experience discomfort, with denuded bone, pain, swelling, suppuration and the formation of a cutaneous fistula or pathological fracture (5).

Treatment must aim to eliminate the pain, control infection and avoid or reduce the spread of ORN. Conservative management is recommended in the early stages, and classically comprises antibiotics, antiseptics and hyperbaric oxygen therapy $(\mathrm{HBO})$ - the latter currently being the subject of considerable controversy (6). Surgery has been used in more evolved stages of ORN, characteri-zed by extensive osteolytic areas, sequestration, cutaneous fistulas or pathological fractures. Ablation surgery of the lesion is first carried out, followed by reconstructive surgery $(7,8)$. In the most advanced phases it proves more difficult to treat and contain progression of the lesions.

Based on a literature review, the present study proposes a new clinical systematization of ORN, placing priority on prevention and early treatment of the lesions, with a view to securing the most effective management possible. In turn, new treatment modalities for ORN are presented.

\section{Literature Review}

\section{-Etiopathogenesis and risk factors}

Radiotherapy appears to cause ORN because it affects the small blood vessels of bone, inducing inflammation (endarteritis), which favors the generation of small thrombi that obliterate the vascular lumen and thus interrupt tissue perfusion (Figure 1). Likewise, RT produces an increase in free radicals and alters collagen synthesis. The bone loses its normal cellularity and undergoes fibrosisatrophy with impairment of its repair and remodeling capacity (4). Under such conditions even minimal external trauma causes ulceration, facilitating contamination and infection, and thus favoring bone necrosis. According to Marx (3), progressive hypoxia, hypovascularization and

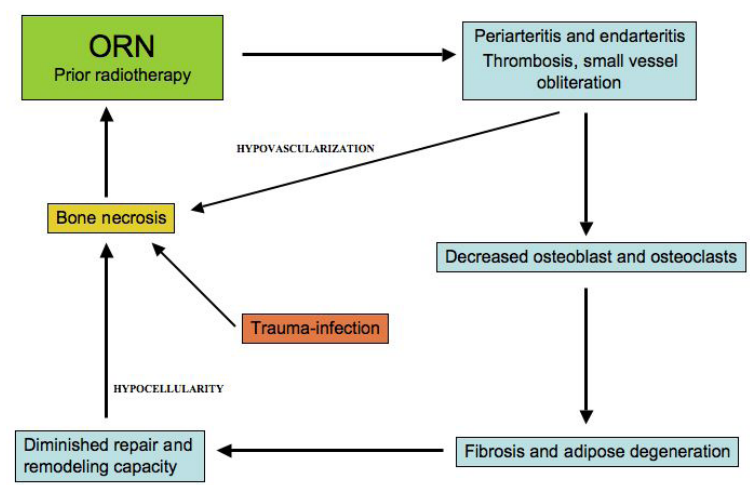

Fig. 1. Algorithm of osteoradionecrosis physiopathology. hypocellularity are observed in the affected bone. However, this metabolic-pathological situation is influenced by a range of triggering and risk factors that have been identified and described in the literature $(4,5,9)$. In general, the risk factors may be related to the tumor, the patient, or the radiotherapy provided.

The tumor-dependent factors fundamentally comprise the anatomical location of the growth, the nearness or degree of involvement of neighboring bone structures, and the tumor stage and size. The patient-dependent factors in turn particularly refer to post-radiotherapy extractions and oral surgery such as osteotomy or mandibular resection, performed before RT. In fact, ORN without prior surgery has been shown to be extremely rare $(2.7 \%$ incidence after 5 years). Other influencing factors are the presence of periodontitis, poor oral hygiene, and previous irradiation of the field (10).

Alcohol consumption and smoking are very important clinical factors, and are much more deleterious in the context of ORN than in maxillary osteonecrosis due to treatment with bisphosphonates, as described by Bagán et al. (11).

Lastly, the radiotherapy-dependent risk factors are the total dose delivered (with increased effects at >65 Gy), dose fractionation, the type of RT used (brachytherapy or external irradiation), and the size of the irradiated area. All these factors capable of increasing the incidence of ORN must be taken into account in order to prevent the development of such lesions (12).

-Clinical and diagnostic considerations

The symptoms of ORN can manifest months or years after irradiation of the patient. In some cases the lesions are discovered in the course of visual inspection of the oral cavity, or due to discomfort experienced in a given area of the mouth. The most commonly affected location is the mandible $(80 \%)$, and in the premolar and molar regions. This appears to be attributable to the increased bone density in these zones, and to their scant collateral circulation compared with the upper maxilla (13).

The lesions manifest as an ulceration, with the exposure of rough and necrotic bone. While clinically the lesions appear small, imaging techniques such as computed tomography (CT) reveal a larger lesion size (2). Overinfection in turn causes pain, and patients may experience dysesthesia in certain zones, as well as chewing or swallowing difficulties (13). The impacting of food in the lesions can give rise to intense halitosis.

The disease tends to take a chronic and progressing course, alternating periods without symptoms and periods characterized by pain - particularly when the lesions become overinfected. The symptoms depend on the location and extent of bone damage (14). In more advanced stages of ORN it is possible to observe bone sequestration, trismus, intense pain, swelling, cutaneous fistulas and pathological mandibular fractures. 
The diagnosis is usually based on the clinical findings and on the patient history, with confirmation being provided by the radiological study and biopsy findings (1-3). The imaging techniques used to assess the extent of osteolysis comprise panoramic X-rays and CT (15).

ORN and bisphosphonate-induced maxillary osteonecrosis may be clinically indistinguishable in the absence of knowledge of the patient antecedents. Nevertheless, Bagán et al. (11), in a study of 20 cases of ORN and 53 cases of maxillary osteonecrosis, reported a series of important differences. Firstly, males were more often affected than females in situations of ORN compared with bisphosphonate-induced maxillary osteonecrosis ( $80 \%$ versus $37.7 \%$ ), and toxic habits such as alcohol abuse and smoking had a greater influence in ORN (smoking 60\%, alcohol 40\%). Likewise, spontaneous presentations were more frequent in ORN (50\%), and the incidence of mandibular fractures (20\%) and cutaneous fistulas (35\%) was also greater in ORN.

\section{-Prevention}

Preventive measures must be evaluated with a view to reducing the risk or severity of ORN. Deficient dental hygiene and septic mouth have been shown to increase the risk of osteoradionecrosis. Likewise, ORN is three times less frequent in edentulous patients than in patients who retain their teeth, possibly as a result of the trauma associated with the need for extractions after irradiation, and the greater number of germs present (16).

Before treatment, a thorough dental exploration is indicated, evaluating those teeth with a poor prognosis due to caries, periodontal disease, or with latent infections (17). Repair should be limited to those teeth that are truly amenable to restoration and which have adequate chances for survival. In these cases extractions should be made at least 2-3 weeks before RT. In the case of retained teeth, this period should be even longer (18).

Another important consideration after RT for lessening the risk of ORN is good fitting, support and stability of removable dentures, avoiding points of excessive pressure that may give rise to pressure ulcers.

\section{-Therapeutic considerations}

To date, the clinical management of ORN has been complex and unsatisfactory. Conservative treatment can be considered in the early stages, when ORN is characterized by a single small lesion. In such situations involving a localized and early-stage lesion, local care and medication (antibiotics) can resolve over half of all cases (17). However, more aggressive surgical treatment is required in more advanced stages.

A separate question is the advisability to use or not use hyperbaric oxygen therapy, the results of which have been the center of considerable controversy and debate. Since its use was suggested for treating ORN, the results of hyperbaric oxygen therapy appear to have been contradictory and scantly convincing (5). The tech- nique involves oxygen perfusion of the tissues, which exerts a stimulatory effect upon repair processes such as osteogenesis. In addition, hyperbaric oxygen therapy improves angiogenesis by increasing the expression of growth factors such as vascular endothelial growth factor (VEGF). However, overinfection with Actinomyces can worsen these conditions $(5,6)$.

Based on the studies published to date, it seems that the inconveniences of hyperbaric oxygen therapy outweigh its advantages. In advanced or refractory cases of ORN, only surgical treatment can be prescribed. In addition, oxygen therapy must be avoided when tumor recurrence or residual tumor tissue is suspected (6). Some authors only recommend the technique in the case of important infection, or when using vascularized free grafts (5).

Medical treatment must be provided early in order to secure good results, at a time when the lesions are still asymptomatic and stable. Irrigation of the lesion with an antiseptic such as chlorhexidine is indicated at this point, with improvements in oral hygiene and the possibility of performing careful curettage or debridement of the exposed area. The pain is treated with analgesics, and antibiotic use is indicated in the case of lesion overinfection. In the presence of important infection, culture and an antibiogram are indicated, though broad spectrum treatment with amoxicillin / clavulanic acid and fluoroquinolone has yielded good results (5). Chronic secondary infection with Actinomyces is refractory to treatment.

When the lesions are extensive and important, with persistent pain, trismus, involvement of the internal cortical layer, cutaneous fistulas or pathological fractures, surgery is required in order to remove all the affected tissue, followed by reconstructive surgery in a later stage (5).

The greatest advances in the surgical management of ORN have been made in reconstructive surgery, replacing the necrotic bone with bone grafts and a regional vascularized flap or with free grafts capable of restoring mandibular continuity with adequate bone volume and quality, sufficient crest height and integrity of the soft tissues (17).

\section{-New therapeutic possibilities}

New treatment possibilities have recently been proposed, though not all of them are supported by the desirable level of scientific evidence. In this context, ultrasound has been suggested with the purpose of promoting angiogenesis, with a direct vasodilatory effect, inducing the synthesis of proteins and cytokines, and stimulating the production of nitrous oxide and prostaglandin E2 on the part of the osteoblasts and fibroblasts (5).

Likewise, techniques such as bone distraction have been proposed to repair previously resected areas, inducing progressive expansion of quality bone and neovascularization in the zone (19). 
In turn, the use of certain biomolecules has been suggested with the aim of directly inducing osteogenesis and thus regenerating the affected areas. These molecules comprise bone growth factors such as bFGF and morphogenetic proteins such as bmp-1 and rhbmp2 (5). However, the studies have been carried out in animal models, without clinical trials or acceptable levels of evidence.

Of all the new therapeutic models, the application of antioxidant agents has offered the best results to date, in the context of controlled clinical trials. Of these antioxidant agents, pentoxifylline (PTX) derived from methylxanthine produces an anti-tumor necrosis factoralpha effect, facilitates microcirculation, and inhibits the inflammatory mechanisms, fibroblast proliferation and the formation of extracellular matrix (20).

Tocopherol (vitamin E) protects the cell membrane against peroxidation, and partially inhibits expression of the procollagen gene and TGF- $\beta 1$ (20).

A synergic effect has been observed between PTX and tocopherol in the treatment of ORN. These are moreover accessible, well tolerated and safe drugs.

Delanian et al. (20), in the context of prolonged treatment (mean $=16 \pm 9$ months) of 54 patients with ORN involving a PTX dose of $800 \mathrm{mg} /$ day and vitamin E 1000 IU/day (5 days a week), recorded an $89 \%$ reduction in bone exposure after 12 months, and an even greater reduction over longer periods of treatment.

\section{Discussion}

Osteoradionecrosis (ORN) is characterized by delayed bone repair secondary to damage caused by radiotherapy (RT). The mean incidence of the disorder is $10 \%$, and it is particularly seen after traumatisms in the form of dental extractions - manifesting between 6 months and 5 years after radiotherapy ( $90 \%$ of the lesions being located in the mandible) (3).

The clinical management of ORN is difficult and normally comprises medical care, the avoidance of toxic habits, improvement of dental hygiene, the control of infections with antibiotics and antiseptics, and removal of the necrotic tissue with more aggressive surgery once complications have appeared (pathological fractures). In addition, many clinical guides mention the possibility of employing hyperbaric oxygen therapy as a coadjuvant measure, though its use is controversial (6).

In any case, no general consensus-based clinico-therapeutic protocol has been established to deal with this serious disorder, despite the experience gained in a similar (though not identical) clinical situation in the form of maxillary osteonecrosis secondary to bisphosphonate therapy. Ruggiero et al. in 2006 (21), and Bagán et al. (22) in 2009, proposed a new clinical classification for maxillary osteonecrosis, based on three evolutive stages - the second stage being subdivided into two substages according to whether the discomfort improves with antibiotic treatment or not. This classification has the advantage of establishing clinical stages that help define more effective treatment strategies, and on the basis of the literature review carried out in this study, it could be used for the management of RT-induced ORN.

The aim is to diagnose the condition in the earliest stage possible. Knowing that the patient has undergone radiotherapy, we should be alerted to detect bone exposure or excessively prolonged socket healing when conducting posterior controls or when having to perform extractions. An early diagnosis is important, since what was previously taken to represent the classical manifestation of ORN (intense pain, swelling, cutaneous fistula or fracture) is presently considered to represent a very advanced stage of the disease.

Thus, as suggested by some authors, it is very important to diagnose the condition in the first days of bone exposure $(5,17)$ - this being the ideal moment for starting treatment (Table 1).

In the early stage of ORN all mucosal irritants should be suppressed (smoking, alcohol, dentures), oral hygiene should be optimized, and $0.2 \%$ chlorhexidine rinses should be applied to the lesions 3-4 times a day.

Controlled clinical trials are needed to assess the efficacy of PTX and vitamin E treatment during this early period (20).

In a second stage, when pain develops, the above measures should be continued, though adding antibiotics such as amoxicillin / clavulanic acid and ciprofloxacin. Once the discomfort has disappeared, the same measures as before are resumed. However, if the pain and discomfort persist, minor surgical interventions can be carried out, such as curettage of the lesion margins or debridement of the exposed area, provided the lesions are small $(<1 \mathrm{~cm}$ in the anterior zone and $<0.5 \mathrm{~cm}$ in posterior sectors) and osteolysis does not reach the external cortical layer (17).

In a third stage, when infection becomes continuous or the extent of osteolysis is considerable, pain proves intense, or fistulization and suppuration or fractures occur, more radical ablation surgery is needed to remove the entire osteolytic zone, leaving vital and vascularized bone margins. Posteriorly, reconstructive surgery can be carried out using pediculate or vascularized free grafts with bone grafting $(5,17)$. Although the treatment of ORN is complex, widely accepted criteria should be established to reduce the advanced complications of osteoradionecrosis and improve the quality of life of these patients. 
Table 1. Treatment of osteoradionecrosis according to the clinical course of the disease.

\begin{tabular}{|rl|}
\hline 1.EARLY STAGE \\
\hline No discomfort associated with the lesion, wich has been present for only a short period of time \\
\hline 1. & Periodic follow-up of the irradiated patient: every 3 months during the first year, and then every 6 months. \\
\hline 2. & Avoid oral mucosal irritants (smoking, alcohol, removable dentures). \\
\hline 3. & Introduction of good oral hygiene. \\
\hline 4. & Irrigation of the lesions with $0.2 \%$ chlorhexidine ( 3 times/day). \\
\hline 5. & Introduction of pentoxifylline ( $800 \mathrm{mg} /$ day) + vitamin AND (1000 IU/day). \\
\hline 2. INTERMEDIATE STAGE \\
\hline Appearance of pain that may or may not improve with antibiotic treatment \\
\hline 1. & Continued observation of good oral hygiene. \\
\hline 2. & Continued irrigation of the lesions with $0.2 \%$ chlorhexidine. \\
\hline 3. & Antibiotic treatment: amoxicillin / clavulanic acid ( $875 \mathrm{mg} / 3$ times a day) + ciprofloxacin $(500 \mathrm{mg}$ twice \\
& daily), until disappearance of the pain. \\
\hline 4. & If the pain and discomfort persist, scantly invasive oral surgery can be carried out, provided the lesion is \\
& small (0.5-1 cm) and does not invade neighboring structures. \\
\hline 3.ADVANCED STAGE \\
\hline Complications appear in the form of trismus, cutaneous fistula or mandibular fracture \\
\hline 1. & The above measures apply. \\
\hline 2. & Ablative surgery to eliminate the osteolytic areas. \\
\hline 3. & Posterior reconstructive surgery with bone grafts, vascularized flaps or free grafts. \\
\hline
\end{tabular}

\section{References}

References with links to Crossref - DOI

1. Peterson DE, Doerr W, Hovan A, Pinto A, Saunders D, Elting LS, et al. Osteoradionecrosis in cancer patients: the evidence base for treatment-dependent frequency, current management strategies, and future studies. Support Care Cancer. 2010;18:1089-98.

2. McLeod NM, Bater MC, Brennan PA. Management of patients at risk of osteoradionecrosis: results of survey of dentists and oral \& maxillofacial surgery units in the United Kingdom, and suggestions for best practice. Br J Oral Maxillofac Surg. 2010;48:301-4.

3. Marx RE. Osteoradionecrosis: a new concept of its pathophysiology. J Oral Maxillofac Surg. 1983;41:283-8.

4. Chrcanovic BR, Reher P, Sousa AA, Harris M. Osteoradionecrosis of the jaws-a current overview--part 1: Physiopathology and risk and predisposing factors. Oral Maxillofac Surg. 2010;14:3-16.

5. Pitak-Arnnop P, Sader R, Dhanuthai K, Masaratana P, Bertolus C, Chaine A, et al. Management of osteoradionecrosis of the jaws: an analysis of evidence. Eur J Surg Oncol. 2008;34:1123-34.

6. Bennett MH, Feldmeier J, Hampson N, Smee R, Milross C. Hyperbaric oxygen therapy for late radiation tissue injury. Cochrane Database Syst Rev. 2005;3:CD005005.

7. Curi MM, Oliveira dos Santos M, Feher O, Faria JC, Rodrigues ML, Kowalski LP. Management of extensive osteoradionecrosis of the mandible with radical resection and immediate microvascular reconstruction. J Oral Maxillofac Surg. 2007;65:434-8.

8. Buchbinder D, St Hilaire H. The use of free tissue transfer in advanced osteoradionecrosis of the mandible. J Oral Maxillofac Surg. 2006;64:961-4.

9. Oh HK, Chambers MS, Martin JW, Lim HJ, Park HJ. Osteoradionecrosis of the mandible: treatment outcomes and factors influencing the progress of osteoradionecrosis. J Oral Maxillofac Surg. 2009;67:1378-86.

10. Jereczek-Fossa BA, Orecchia R. Radiotherapy-induced mandibular bone complications. Cancer Treat Rev. 2002;28:65-74.

11. Bagan JV, Jiménez Y, Hernández S, Murillo J, Díaz JM, Poveda R, et al. Osteonecrosis of the jaws by intravenous bisphosphonates and osteoradionecrosis: a comparative study. Med Oral Patol Oral Cir Bucal. 2009;14:e616-9.

12. Lee IJ, Koom WS, Lee CG, Kim YB, Yoo SW, Keum KC, et al.
Risk factors and dose-effect relationship for mandibular osteoradionecrosis in oral and oropharyngeal cancer patients. Int J Radiat Oncol Biol Phys. 2009; 75:1084-91.

13. Marx RE, Johnson RP. Studies in the radiobiology of osteoradionecrosis and their clinical significance. Oral Surg Oral Med Oral Pathol. 1987;64:379-90.

14. Thariat J, de Mones E, Darcourt V, Poissonnet G, Marcy PY, Guevara N, et al. Teeth and irradiation: dental care and treatment of osteoradionecrosis after irradiation in head and neck cancer. Cancer Radiother. 2010;14:137-44.

15. Le Stanc E, Vilain D, Baglin AC, Tainturier C. Mandibular lesion differential diagnoses in a patient with a previous history of locally advanced head and neck carcinoma. Clin Nucl Med. 2009;34:435-8. 16. Chrcanovic BR, Reher P, Sousa AA, Harris M. Osteoradionecrosis of the jaws-a current overview--Part 2: dental management and therapeutic options for treatment. Oral Maxillofac Surg. 2010;14:81-95.

17. Madrid C, Abarca M, Bouferrache K. Osteoradionecrosis: an update. Oral Oncol. 2010;46:471-4.

18. Beumer J, Harrison R, Sanders B, Kurrasch M. Osteoradionecrosis: predisposing factors and outcomes of therapy. Head Neck Surg. 1984;6:819-27.

19. González-García R, Rodríguez-Campo FJ, Naval-Gías L, SastrePérez J, Díaz-González FJ. The effect of radiation in distraction osteogenesis for reconstruction of mandibular segmental defects. Br J Oral Maxillofac Surg. 2007;45:314-6.

20. Delanian S, Chatel C, Porcher R, Depondt J, Lefaix JL. Complete restoration of refractory mandibular osteoradionecrosis by prolonged treatment with a pentoxifylline-tocopherol-clodronate combination (PENTOCLO): a phase II trial. Int J Radiat Oncol Biol Phys. 2011;80:832-9.

21. Ruggiero SL, Fantasia J, Carlson E. Bisphosphonate-related osteonecrosis of the jaw: background and guidelines for diagnosis, staging and management. Oral Surg Oral Med Oral Pathol Oral Radiol Endod. 2006;102:433-41.

22. Bagan JV, Jimenez Y, Diaz JM, Murillo J, Sanchis JM, Poveda $\mathrm{R}$, et al. Osteonecrosis of the jaws in intravenous bisphosphonate use: Proposal for a modification of the clinical classification. Oral Oncol. 2009;45:645-6. 